\title{
Combined search for neutrinos from dark matter self-annihilation in the Galactic Center with ANTARES and IceCube
}

A. Albert, ${ }^{1,2}$ M. André, ${ }^{3}$ M. Anghinolfi, ${ }^{4}$ M. Ardid, ${ }^{5}$ J.-J. Aubert,,${ }^{6}$ J. Aublin, ${ }^{7}$ B. Baret, ${ }^{7}$ S. Basa,${ }^{8}$ B. Belhorma, ${ }^{9}$ V. Bertin, ${ }^{6}$ S. Biagi, ${ }^{10}$ M. Bissinger, ${ }^{11}$ J. Boumaaza, ${ }^{12}$ M. Bouta, ${ }^{13}$ M. C. Bouwhuis, ${ }^{14}$ H. Brânzaş, ${ }^{15}$ R. Bruijn,${ }^{14,16}$ J. Brunner, ${ }^{6}$ J. Busto, ${ }^{6}$ A. Capone, ${ }^{17,18}$ L. Caramete, ${ }^{15}$ J. Carr, ${ }^{6}$ S. Celli, ${ }^{17,18,19}$ M. Chabab ${ }^{20}$ T. N. Chau, ${ }^{7}$ R. Cherkaoui El Moursli, ${ }^{12}$

T. Chiarusi, ${ }^{21}$ M. Circella, ${ }^{22}$ A. Coleiro, ${ }^{7}$ M. Colomer, ${ }^{7,23}$ R. Coniglione, ${ }^{10}$ P. Coyle, ${ }^{6}$ A. Creusot, ${ }^{7}$ A. F. Díaz, ${ }^{24}$ A. Deschamps ${ }^{25}$ C. Distefano, ${ }^{10}$ I. Di Palma, ${ }^{17,18}$ A. Domi ${ }^{4,26}$ C. Donzaud ${ }^{7,27}$ D. Dornic, ${ }^{6}$ D. Drouhin,,${ }^{1,2}$ T. Eberl, ${ }^{11}$ N. El Khayati, ${ }^{12}$ A. Enzenhöfer, ${ }^{11,6}$ A. Ettahiri, ${ }^{12}$ P. Fermani, ${ }^{17,18}$ G. Ferrara ${ }^{10}$ F. Filippini, ${ }^{21,28}$ L. Fusco,${ }^{7,28}$ P. Gay, ${ }^{29,7}$ H. Glotin ${ }^{30}$ R. Gozzini, ${ }^{23}$ R. Gracia Ruiz, ${ }^{1}$ K. Graf, ${ }^{11}$ C. Guidi, ${ }^{4,26}$ S. Hallmann, ${ }^{11}$ H. van Haren, ${ }^{31}$ A. J. Heijboer, ${ }^{14}$ Y. Hello, ${ }^{25}$ J. J. Hernández-Rey, ${ }^{23}$ J. Höß1, ${ }^{11}$ J. Hofestädt, ${ }^{11}$ F. Huang, ${ }^{1}$ G. Illuminati, ${ }^{23}$ C. W. James, ${ }^{32}$ M. de Jong ${ }^{14,33}$

P. de Jong, ${ }^{14}$ M. Jongen, ${ }^{14}$ M. Kadler, ${ }^{34}$ O. Kalekin, ${ }^{11}$ U. Katz, ${ }^{11}$ N. R. Khan-Chowdhury, ${ }^{23}$ A. Kouchner, ${ }^{7,35}$

I. Kreykenbohm, ${ }^{36}$ V. Kulikovskiy, ${ }^{4,37}$ R. Lahmann, ${ }^{11}$ R. Le Breton, ${ }^{7}$ D. Lefèvre,${ }^{38}$ E. Leonora, ${ }^{39}$ G. Levi, ${ }^{21,28}$ M. Lincetto, ${ }^{6}$

D. Lopez-Coto, ${ }^{40}$ S. Loucatos, ${ }^{41,7}$ G. Maggi, ${ }^{6}$ J. Manczak ${ }^{23}$ M. Marcelin, ${ }^{8}$ A. Margiotta, ${ }^{21,28}$ A. Marinelli, ${ }^{42,43}$

J. A. Martínez-Mora, ${ }^{5}$ R. Mele ${ }^{44,45}$ K. Melis, ${ }^{14,16}$ P. Migliozzi, ${ }^{44}$ M. Moser, ${ }^{11}$ A. Moussa,${ }^{13}$ R. Muller ${ }^{14}$ L. Nauta, ${ }^{14}$ S. Navas, ${ }^{40}$ E. Nezri, ${ }^{8}$ C. Nielsen, ${ }^{7}$ A. Nuñez-Castiñeyra, ${ }^{6,8}$ B. O’Fearraigh, ${ }^{14}$ M. Organokov, ${ }^{1}$ G. E. Păvălaş, ${ }^{15}$

C. Pellegrino, ${ }^{21,28}$ M. Perrin-Terrin, ${ }^{6}$ P. Piattelli, ${ }^{10}$ C. Poirè,${ }^{5}$ V. Popa ${ }^{15}$ T. Pradier, ${ }^{1}$ N. Randazzo, ${ }^{39}$ S. Reck,,${ }^{11}$

G. Riccobene, ${ }^{10}$ A. Sánchez-Losa, ${ }^{22}$ D. F. E. Samtleben ${ }^{14,33}$ M. Sanguineti ${ }^{4,26}$ P. Sapienza, ${ }^{10}$ F. Schüssler, ${ }^{41}$ M. Spurio, ${ }^{21,28}$ Th. Stolarczyk, ${ }^{41}$ B. Strandberg, ${ }^{14}$ M. Taiuti, ${ }^{4,26}$ Y. Tayalati, ${ }^{12}$ T. Thakore ${ }^{23}$ S. J. Tingay, ${ }^{32}$ A. Trovato, ${ }^{10}$

B. Vallage, ${ }^{41,7}$ V. Van Elewyck, ${ }^{7,35}$ F. Versari, ${ }^{21,28,7}$ S. Viola, ${ }^{10}$ D. Vivolo, ${ }^{44,45}$ J. Wilms, ${ }^{36}$ D. Zaborov, ${ }^{6}$ A. Zegarelli, ${ }^{17,18}$ J. D. Zornoza, ${ }^{23}$ J. Zúñiga, ${ }^{23}$

\section{(ANTARES Collaboration)}

M. G. Aartsen, ${ }^{46}$ M. Ackermann, ${ }^{47}$ J. Adams, ${ }^{46}$ J. A. Aguilar, ${ }^{48}$ M. Ahlers, ${ }^{49}$ M. Ahrens,${ }^{50}$ C. Alispach,${ }^{51}$ K. Andeen,${ }^{52}$ T. Anderson, ${ }^{53}$ I. Ansseau, ${ }^{48}$ G. Anton, ${ }^{54}$ C. Argüelles, ${ }^{55}$ J. Auffenberg, ${ }^{56}$ S. Axani ${ }^{55}$ H. Bagherpour, ${ }^{46}$ X. Bai ${ }^{57}$ A. Balagopal V. ${ }^{58}$ A. Barbano, ${ }^{51}$ S. W. Barwick, ${ }^{59}$ B. Bastian, ${ }^{47}$ V. Baum, ${ }^{60}$ S. Baur, ${ }^{48}$ R. Bay, ${ }^{61}$ J. J. Beatty, ${ }^{62,63}$ K.-H. Becker, ${ }^{64}$ J. Becker Tjus, ${ }^{65}$ S. BenZvi, ${ }^{66}$ D. Berley, ${ }^{67}$ E. Bernardini, ${ }^{47,}{ }^{*}$ D. Z. Besson, ${ }^{68,}$ G. Binder, ${ }^{61,69}$ D. Bindig ${ }^{64}$ E. Blaufuss ${ }^{67}$ S. Blot, ${ }^{47}$ C. Bohm,${ }^{50}$ S. Böser, ${ }^{60}$ O. Botner, ${ }^{70}$ J. Böttcher,${ }^{56}$ E. Bourbeau, ${ }^{49}$ J. Bourbeau ${ }^{71}$ F. Bradascio, ${ }^{47}$ J. Braun, ${ }^{71}$ S. Bron, ${ }^{51}$ J. Brostean-Kaiser, ${ }^{47}$ A. Burgman,${ }^{70}$ J. Buscher, ${ }^{56}$ R. S. Busse, ${ }^{72}$ T. Carver, ${ }^{51}$ C. Chen, ${ }^{73}$ E. Cheung, ${ }^{67}$ D. Chirkin ${ }^{71}$ S. Choi, ${ }^{74}$ B. A. Clark, ${ }^{75}$ K. Clark, ${ }^{76}$ L. Classen, ${ }^{72}$ A. Coleman, ${ }^{77}$ G. H. Collin,${ }^{55}$ J. M. Conrad,${ }^{55}$ P. Coppin, ${ }^{78}$ P. Correa ${ }^{78}$ D. F. Cowen, ${ }^{79,53}$ R. Cross, ${ }^{66}$ P. Dave, ${ }^{73}$ C. De Clercq,${ }^{78}$ J. J. DeLaunay, ${ }^{53}$ H. Dembinski ${ }^{77}$ K. Deoskar, ${ }^{50}$ S. De Ridder ${ }^{80}$ P. Desiati, ${ }^{71}$ K. D. de Vries, ${ }^{78}$ G. de Wasseige, ${ }^{78}$ M. de With, ${ }^{81}$ T. DeYoung, ${ }^{75}$ A. Diaz, ${ }^{55}$ J. C. Díaz-Vélez,${ }^{71}$ H. Dujmovic, ${ }^{58}$ M. Dunkman, ${ }^{33}$ E. Dvorak, ${ }^{57}$ B. Eberhardt, ${ }^{71}$ T. Ehrhardt, ${ }^{60}$ P. Eller, ${ }^{53}$ R. Engel,${ }^{58}$ P. A. Evenson, ${ }^{77}$ S. Fahey ${ }^{71}$ A. R. Fazely, ${ }^{82}$ J. Felde,${ }^{67}$ K. Filimonov ${ }^{61}$ C. Finley,${ }^{50}$ D. Fox ${ }^{79}$ A. Franckowiak, ${ }^{47}$ E. Friedman, ${ }^{67}$ A. Fritz, ${ }^{60}$ T. K. Gaisser, ${ }^{77}$ J. Gallagher, ${ }^{83}$ E. Ganster, ${ }^{56}$ S. Garrappa, ${ }^{47}$ L. Gerhardt, ${ }^{69}$ K. Ghorbani, ${ }^{71}$ T. Glauch, ${ }^{84}$ T. Glüsenkamp, ${ }^{54}$ A. Goldschmidt ${ }^{69}$ J. G. Gonzalez, ${ }^{77}$ D. Grant, ${ }^{75}$ T. Grégoire, ${ }^{53}$ Z. Griffith ${ }^{71}$ S. Griswold, ${ }^{66}$ M. Günder, ${ }^{56}$ M. Gündüz, ${ }^{65}$ C. Haack, ${ }^{56}$ A. Hallgren, ${ }^{70}$ R. Halliday,${ }^{75}$ L. Halve,${ }^{56}$ F. Halzen, ${ }^{71}$ K. Hanson, ${ }^{71}$ A. Haungs, ${ }^{58}$ D. Hebecker, ${ }^{81}$ D. Heereman, ${ }^{48}$ P. Heix,${ }^{56}$ K. Helbing, ${ }^{64}$ R. Hellauer, ${ }^{67}$ F. Henningsen,${ }^{84}$ S. Hickford, ${ }^{64}$ J. Hignight ${ }^{85}$ G. C. Hill, ${ }^{86}$ K. D. Hoffman ${ }^{67}$ R. Hoffmann, ${ }^{64}$ T. Hoinka ${ }^{87}$ B. Hokanson-Fasig, ${ }^{71}$ K. Hoshina ${ }^{71,}$ M. Huber, ${ }^{84}$ T. Huber, ${ }^{58,47}$ K. Hultqvist, ${ }^{50}$ M. Hünnefeld, ${ }^{87}$ R. Hussain, ${ }^{71}$ S. In ${ }^{74}$ N. Iovine $\odot,{ }^{48}$ A. Ishihara ${ }^{88}{ }^{88}$ M. Jansson, ${ }^{50}$ G. S. Japaridze, ${ }^{89}$ M. Jeong ${ }^{74}$ K. Jero ${ }^{71}$ B. J. P. Jones, ${ }^{90}$ F. Jonske, ${ }^{56}$ R. Joppe, ${ }^{56}$ D. Kang, ${ }^{58}$ W. Kang, ${ }^{74}$ A. Kappes, ${ }^{72}$ D. Kappesser, ${ }^{60}$ T. Karg, ${ }^{47}$ M. Karl, ${ }^{84}$ A. Karle, ${ }^{71}$ M. Kauer, ${ }^{71}$ M. Kellermann, ${ }^{56}$ J. L. Kelley, ${ }^{71}$ A. Kheirandish, ${ }^{53}$ J. Kim ${ }_{75}{ }^{74}$ T. Kintscher, ${ }^{47}$ J. Kiryluk, ${ }^{91}$ T. Kittler, ${ }^{54}$ S. R. Klein, ${ }^{61,69}$ R. Koirala, ${ }^{77}$ H. Kolanoski, ${ }^{81}$ L. Köpke, ${ }^{60}$ C. Kopper, ${ }^{75}$ S. Kopper, ${ }^{92}$

D. J. Koskinen, ${ }^{49}$ M. Kowalski, ${ }^{81,47}$ K. Krings, ${ }^{84}$ G. Krückl,${ }^{60}$ N. Kulacz, ${ }^{85}$ N. Kurahashi, ${ }^{93}$ A. Kyriacou, ${ }^{86}$

J. L. Lanfranchi, ${ }^{53}$ M. J. Larson, ${ }^{67}$ F. Lauber, ${ }^{64}$ J. P. Lazar, ${ }^{71}$ K. Leonard, ${ }^{71}$ A. Leszczyńska, ${ }^{58}$ Q. R. Liu ${ }^{71}$ E. Lohfink, ${ }^{60}$ C. J. Lozano Mariscal, ${ }^{72}$ L. Lu, ${ }^{88}$ F. Lucarelli, ${ }^{51}$ A. Ludwig, ${ }^{94}$ J. Lünemann, ${ }^{78}$ W. Luszczak, ${ }^{71}$ Y. Lyu, ${ }^{61,69}$ W. Y. Ma, ${ }^{47}$ J. Madsen, ${ }^{95}$ K. B. M. Mahn, ${ }^{75}$ Y. Makino, ${ }^{88}$ P. Mallik, ${ }^{56}$ K. Mallot, ${ }^{71}$ S. Mancina, ${ }^{71}$ I. C. Mariş, ${ }^{48}$ R. Maruyama, ${ }^{96}$ K. Mase, ${ }^{88}$ R. Maunu, ${ }^{67}$ F. McNally, ${ }^{97}$ K. Meagher, ${ }^{71}$ M. Medici, ${ }^{49}$ A. Medina, ${ }^{63}$ M. Meier, ${ }^{87}$ S. Meighen-Berger ${ }^{84}$ G. Merino, ${ }^{71}$ T. Meures, ${ }^{48}$ J. Micallef, ${ }^{75}$ D. Mockler ${ }^{48}$ G. Momenté, ${ }^{60}$ T. Montaruli, ${ }^{51}$ R. W. Moore, ${ }^{85}$ R. Morse ${ }^{71}$ M. Moulai,${ }^{55}$ P. Muth, ${ }^{56}$ R. Nagai ${ }^{88}$ U. Naumann, ${ }^{64}$ G. Neer ${ }^{75}$ L. V. Nguyễn ${ }^{75}$ H. Niederhausen, ${ }^{84}$ M. U. Nisa ${ }^{75}$ S. C. Nowicki, ${ }^{75}$ D. R. Nygren, ${ }^{69}$ A. Obertacke Pollmann, ${ }^{64}$ M. Oehler, ${ }^{58}$ A. Olivas, ${ }^{67}$ A. O’Murchadha, ${ }^{48}$ E. O’'Sullivan, ${ }^{50}$ T. Palczewski, ${ }^{61,69}$ H. Pandya, ${ }^{77}$ D. V. Pankova, ${ }^{53}$ N. Park, ${ }^{71}$ P. Peiffer, ${ }^{60}$ C. Pérez de los Heros,${ }^{70}$ S. Philippen, ${ }^{56}$ 
D. Pieloth, ${ }^{87}$ S. Pieper, ${ }^{64}$ E. Pinat, ${ }^{48}$ A. Pizzuto, ${ }^{71}$ M. Plum, ${ }^{52}$ A. Porcelli, ${ }^{80}$ P. B. Price, ${ }^{61}$ G. T. Przybylski, ${ }^{69}$ C. Raab ${ }^{48}$ A. Raissi, ${ }^{46}$ M. Rameez, ${ }^{49}$ L. Rauch, ${ }^{47}$ K. Rawlins, ${ }^{98}$ I. C. Rea, ${ }^{84}$ A. Rehman, ${ }^{77}$ R. Reimann, ${ }^{56}$ B. Relethford, ${ }^{93}$ M. Renschler, ${ }^{58}$ G. Renzi, ${ }^{48}$ E. Resconi, ${ }^{84}$ W. Rhode, ${ }^{87}$ M. Richman, ${ }^{93}$ S. Robertson, ${ }^{69}$ M. Rongen, ${ }^{56}$ C. Rott, ${ }^{74}$ T. Ruhe, ${ }^{87}$ D. Ryckbosch, ${ }^{80}$ D. Rysewyk Cantu, ${ }^{75}$ I. Safa, ${ }^{71}$ S. E. Sanchez Herrera, ${ }^{75}$ A. Sandrock, ${ }^{87}$ J. Sandroos, ${ }^{60}$ M. Santander, ${ }^{92}$ S. Sarkar, ${ }^{99}$ S. Sarkar, ${ }^{85}$ K. Satalecka, ${ }^{47}$ M. Schaufel, ${ }^{56}$ H. Schieler, ${ }^{58}$ P. Schlunder, ${ }^{87}$ T. Schmidt, ${ }^{67}$ A. Schneider, ${ }^{71}$ J. Schneider, ${ }^{54}$ F. G. Schröder, ${ }^{58,77}$ L. Schumacher, ${ }^{56}$ S. Sclafani, ${ }^{93}$ D. Seckel, ${ }^{77}$ S. Seunarine, ${ }^{95}$ S. Shefali, ${ }^{56}$ M. Silva, ${ }^{71}$ R. Snihur, ${ }^{71}$ J. Soedingrekso, ${ }^{87}$ D. Soldin, ${ }^{77}$ M. Song, ${ }^{67}$ G. M. Spiczak, ${ }^{95}$ C. Spiering, ${ }^{47}$ J. Stachurska, ${ }^{47}$ M. Stamatikos, ${ }^{63}$ T. Stanev, ${ }^{77}$ R. Stein, ${ }^{47}$ J. Stettner, ${ }^{56}$ A. Steuer, ${ }^{60}$ T. Stezelberger, ${ }^{69}$ R. G. Stokstad, ${ }^{69}$ A. Stöß1, ${ }^{88}$ N. L. Strotjohann, ${ }^{47}$ T. Stürwald, ${ }^{56}$ T. Stuttard, ${ }^{49}$ G. W. Sullivan, ${ }^{67}$ I. Taboada, ${ }^{73}$ F. Tenholt, ${ }^{65}$ S. Ter-Antonyan, ${ }^{82}$ A. Terliuk, ${ }^{47}$ S. Tilav, ${ }^{77}$ K. Tollefson, ${ }^{75}$ L. Tomankova, ${ }^{65}$ C. Tönnis, ${ }^{100}$ S. Toscano, ${ }^{48}$ D. Tosi, ${ }^{71}$ A. Trettin, ${ }^{47}$ M. Tselengidou, ${ }^{54}$ C. F. Tung, ${ }^{73}$ A. Turcati, ${ }^{84}$ R. Turcotte, ${ }^{58}$ C. F. Turley, ${ }^{53}$ B. Ty, ${ }^{71}$ E. Unger, ${ }^{70}$ M. A. Unland Elorrieta, ${ }^{72}$ M. Usner, ${ }^{47}$ J. Vandenbroucke, $^{71}$ W. Van Driessche, ${ }_{86}^{80}$ D. van Eijk, ${ }_{56}^{71}$ N. van Eijndhoven, ${ }^{78}$ J. van Santen, ${ }^{47}$ S. Verpoest, ${ }_{85}^{80}$ M. Vraeghe, ${ }_{53}^{80}$ C. Walck, ${ }^{50}$ A. Wallace, ${ }^{86}$ M. Wallraff, ${ }^{56}$ N. Wandkowsky, ${ }^{71}$ T. B. Watson, ${ }^{90}$ C. Weaver, ${ }^{85}$ A. Weindl, ${ }^{58}$ M. J. Weiss, ${ }^{53}$ J. Weldert, ${ }^{60}$ C. Wendt, ${ }^{71}$ J. Werthebach, ${ }^{71}$ B. J. Whelan, ${ }^{86}$ N. Whitehorn, ${ }^{94}$ K. Wiebe, ${ }^{60}$ C. H. Wiebusch, ${ }^{56}$ L. Wille, $^{71}$ D. R. Williams, ${ }^{92}$ L. Wills, ${ }^{93}$ M. Wolf, ${ }^{84}$ J. Wood, ${ }^{71}$ T. R. Wood, ${ }^{85}$ K. Woschnagg, ${ }^{61}$ G. Wrede, ${ }^{54}$ D. L. Xu, ${ }^{71}$ X. W. Xu, ${ }^{82}$ Y. Xu, ${ }^{91}$ J. P. Yanez, ${ }^{85}$ G. Yodh, ${ }^{59}$ S. Yoshida, ${ }^{88}$ T. Yuan, ${ }^{71}$ and M. Zöcklein ${ }^{56}$

\section{(IceCube Collaboration)}

${ }^{1}$ Université de Strasbourg, CNRS, IPHC UMR 7178, F-67000 Strasbourg, France

${ }^{2}$ Université de Haute Alsace, F-68200 Mulhouse, France

${ }^{3}$ Technical University of Catalonia, Laboratory of Applied Bioacoustics, Rambla Exposició, 08800 Vilanova i la Geltrú, Barcelona, Spain

${ }^{4}$ INFN - Sezione di Genova, Via Dodecaneso 33, 16146 Genova, Italy

${ }^{5}$ Institut d'Investigació per a la Gestió Integrada de les Zones Costaneres (IGIC) - Universitat Politècnica de València., C/ Paranimf 1, 46730 Gandia, Spain

${ }^{6}$ Aix Marseille Univ, CNRS/IN2P3, CPPM, Marseille, France

${ }^{7}$ APC, Université de Paris, CNRS, Astroparticule et Cosmologie, F-75013 Paris, France

${ }^{8}$ Aix Marseille Univ, CNRS, CNES, LAM, Marseille, France

${ }^{9}$ National Center for Energy Sciences and Nuclear Techniques, B.P.1382, R. P.10001 Rabat, Morocco

${ }^{10}$ INFN - Laboratori Nazionali del Sud (LNS), Via S. Sofia 62, 95123 Catania, Italy

${ }^{11}$ Friedrich-Alexander-Universität Erlangen-Nürnberg, Erlangen Centre for Astroparticle Physics, Erwin-Rommel-Str. 1, 91058 Erlangen, Germany

${ }^{12}$ University Mohammed $V$ in Rabat, Faculty of Sciences, 4 av. Ibn Battouta, B.P. 1014, R.P. 10000 Rabat, Morocco

${ }^{13}$ University Mohammed I, Laboratory of Physics of Matter and Radiations, B.P.717, Oujda 6000, Morocco

${ }^{14}$ Nikhef, Science Park, Amsterdam, The Netherlands

${ }^{15}$ Institute of Space Science, RO-077125 Bucharest, Măgurele, Romania

${ }^{16}$ Universiteit van Amsterdam, Instituut voor Hoge-Energie Fysica, Science Park 105, 1098 XG Amsterdam, The Netherlands

${ }^{17}$ INFN - Sezione di Roma, P.le Aldo Moro 2, 00185 Roma, Italy

${ }^{18}$ Dipartimento di Fisica dell'Università La Sapienza, P.le Aldo Moro 2, 00185 Roma, Italy

${ }^{19}$ Gran Sasso Science Institute, Viale Francesco Crispi 7, 00167 L'Aquila, Italy

${ }^{20}$ LPHEA, Faculty of Science - Semlali, Cadi Ayyad University, P.O.B. 2390, Marrakech, Morocco

${ }^{21}$ INFN - Sezione di Bologna, Viale Berti-Pichat 6/2, 40127 Bologna, Italy

${ }^{22}$ INFN - Sezione di Bari, Via E. Orabona 4, 70126 Bari, Italy

${ }^{23}$ IFIC - Instituto de Física Corpuscular (CSIC - Universitat de València), cl Catedrático José Beltrán, 2 E-46980 Paterna, Valencia, Spain

${ }^{24}$ Department of Computer Architecture and Technology/CITIC, University of Granada, 18071 Granada, Spain

${ }^{25}$ Géoazur, UCA, CNRS, IRD, Observatoire de la Côte d'Azur, Sophia Antipolis, France

${ }^{26}$ Dipartimento di Fisica dell'Università, Via Dodecaneso 33, 16146 Genova, Italy

${ }^{27}$ Université Paris-Sud, 91405 Orsay Cedex, France

${ }^{28}$ Dipartimento di Fisica e Astronomia dell'Università, Viale Berti Pichat 6/2, 40127 Bologna, Italy

${ }^{29}$ Laboratoire de Physique Corpusculaire, Clermont Université, Université Blaise Pascal,

CNRS/IN2P3, BP 10448, F-63000 Clermont-Ferrand, France 
${ }^{30}$ LIS, UMR Université de Toulon, Aix Marseille Université, CNRS, 83041 Toulon, France

${ }^{31}$ Royal Netherlands Institute for Sea Research (NIOZ) and Utrecht University, Landsdiep 4, 1797 SZ 't Horntje (Texel), the Netherlands

${ }^{32}$ International Centre for Radio Astronomy Research - Curtin University, Bentley, Western Australia 6102, Australia

${ }^{33}$ Huygens-Kamerlingh Onnes Laboratorium, Universiteit Leiden, The Netherlands

${ }^{34}$ Institut für Theoretische Physik und Astrophysik, Universität Würzburg, Emil-Fischer Str. 31, 97074 Würzburg, Germany

${ }^{35}$ Institut Universitaire de France, 75005 Paris, France

${ }^{36}$ Dr. Remeis-Sternwarte and ECAP, Friedrich-Alexander-Universität Erlangen-Nürnberg, Sternwartstr. 7, 96049 Bamberg, Germany

${ }^{37}$ Moscow State University, Skobeltsyn Institute of Nuclear Physics, Leninskie gory, 119991 Moscow, Russia

${ }^{38}$ Mediterranean Institute of Oceanography (MIO), Aix-Marseille University, 13288, Marseille, Cedex 9, France;

Université du Sud Toulon-Var, CNRS-INSU/IRD UM 110, 83957, La Garde Cedex, France

${ }^{39}$ INFN - Sezione di Catania, Via S. Sofia 64, 95123 Catania, Italy

${ }^{40}$ Departamento de Física Teórica y del Cosmos \& C.A.F.P.E., University of Granada, 18071 Granada, Spain

${ }^{41}$ IRFU, CEA, Université Paris-Saclay, F-91191 Gif-sur-Yvette, France

${ }^{42}$ INFN - Sezione di Pisa, Largo B. Pontecorvo 3, 56127 Pisa, Italy

${ }^{43}$ Dipartimento di Fisica dell'Università, Largo B. Pontecorvo 3, 56127 Pisa, Italy

${ }^{44}$ INFN - Sezione di Napoli, Via Cintia 80126 Napoli, Italy

${ }^{45}$ Dipartimento di Fisica dell'Università Federico II di Napoli, Via Cintia 80126, Napoli, Italy

${ }^{46}$ Department of Physics and Astronomy, University of Canterbury, Private Bag 4800, Christchurch, New Zealand

${ }^{47}$ DESY, D-15738 Zeuthen, Germany

${ }^{48}$ Université Libre de Bruxelles, Science Faculty CP230, B-1050 Brussels, Belgium

${ }^{49}$ Niels Bohr Institute, University of Copenhagen, DK-2100 Copenhagen, Denmark

${ }^{50}$ Oskar Klein Centre and Department of Physics, Stockholm University, SE-10691 Stockholm, Sweden

${ }^{51}$ Département de physique nucléaire et corpusculaire, Université de Genève, CH-1211 Genève, Switzerland

${ }^{52}$ Department of Physics, Marquette University, Milwaukee, Wisconsin 53201, USA

${ }^{53}$ Department of Physics, Pennsylvania State University, University Park, Pennsylvania 16802, USA

${ }^{54}$ Erlangen Centre for Astroparticle Physics, Friedrich-Alexander-Universität Erlangen-Nürnberg, D-91058 Erlangen, Germany

${ }^{55}$ Department of Physics, Massachusetts Institute of Technology, Cambridge, Massachusetts 02139, USA

${ }^{56}$ III. Physikalisches Institut, RWTH Aachen University, D-52056 Aachen, Germany

${ }^{57}$ Physics Department, South Dakota School of Mines and Technology, Rapid City, South Dakota 57701, USA

${ }^{58}$ Karlsruhe Institute of Technology, Institut für Kernphysik, D-76021 Karlsruhe, Germany

${ }^{59}$ Department of Physics and Astronomy, University of California, Irvine, California 92697, USA

${ }^{60}$ Institute of Physics, University of Mainz, Staudinger Weg 7, D-55099 Mainz, Germany

${ }^{61}$ Department of Physics, University of California, Berkeley, California 94720, USA

${ }^{62}$ Department of Astronomy, Ohio State University, Columbus, Ohio 43210, USA

${ }^{63}$ Department of Physics and Center for Cosmology and Astro-Particle Physics, Ohio State University, Columbus, Ohio 43210, USA

${ }^{64}$ Department of Physics, University of Wuppertal, D-42119 Wuppertal, Germany

${ }^{65}$ Fakultät für Physik \& Astronomie, Ruhr-Universität Bochum, D-44780 Bochum, Germany

${ }^{66}$ Department of Physics and Astronomy, University of Rochester, Rochester, New York 14627, USA

${ }^{67}$ Department of Physics, University of Maryland, College Park, Maryland 20742, USA

${ }^{68}$ Department of Physics and Astronomy, University of Kansas, Lawrence, Kansas 66045, USA

${ }^{69}$ Lawrence Berkeley National Laboratory, Berkeley, California 94720, USA

${ }^{70}$ Department of Physics and Astronomy, Uppsala University, Box 516, S-75120 Uppsala, Sweden

${ }^{71}$ Department of Physics and Wisconsin IceCube Particle Astrophysics Center, University of Wisconsin, Madison, Wisconsin 53706, USA

${ }^{72}$ Institut für Kernphysik, Westfälische Wilhelms-Universität Münster, D-48149 Münster, Germany

${ }^{73}$ School of Physics and Center for Relativistic Astrophysics, Georgia Institute of Technology, Atlanta, Georgia 30332, USA

${ }^{74}$ Department of Physics, Sungkyunkwan University, Suwon 16419, Korea 


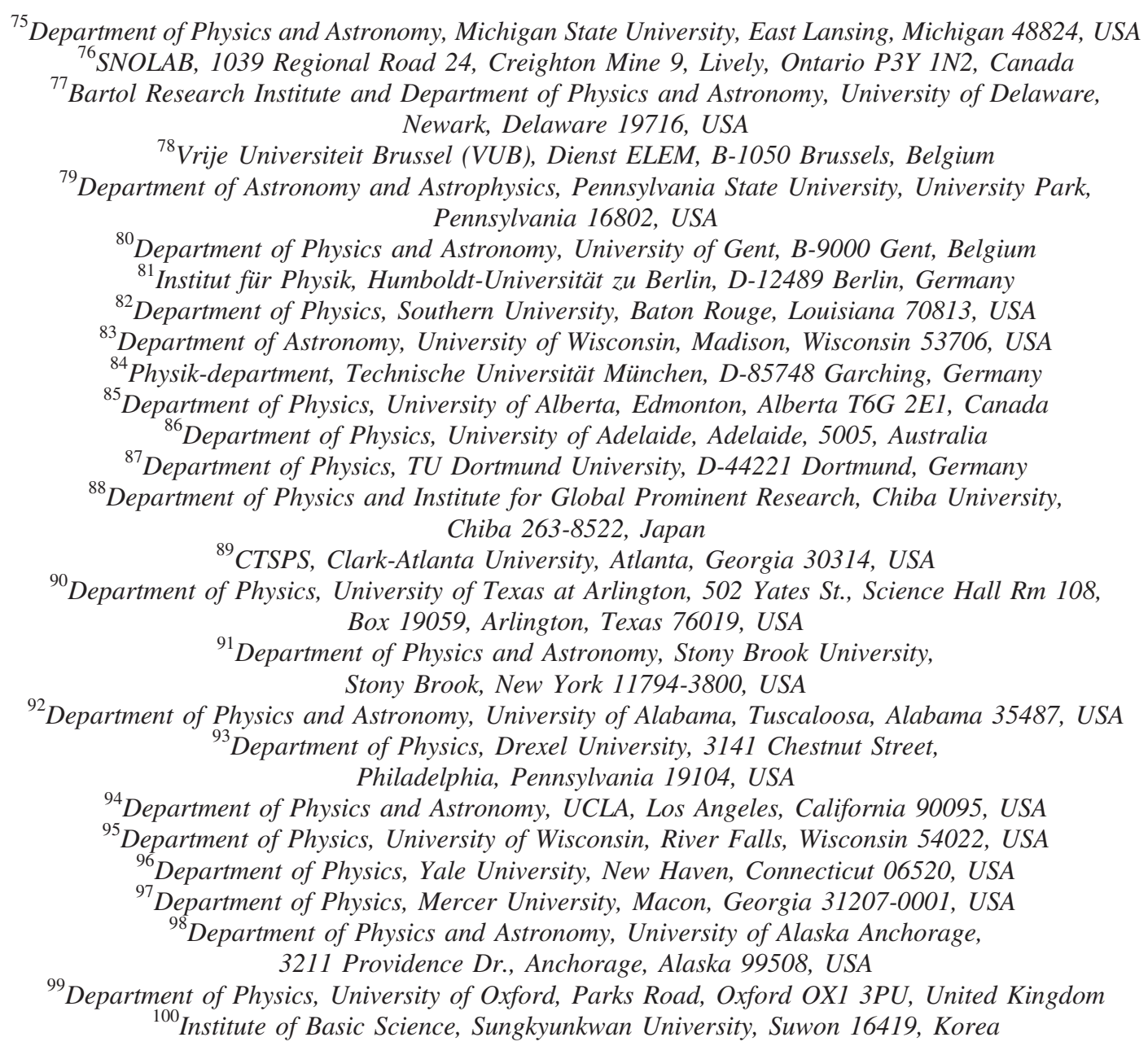

(Received 14 March 2020; accepted 27 August 2020; published 27 October 2020)

We present the results of the first combined dark matter search targeting the Galactic Center using the ANTARES and IceCube neutrino telescopes. For dark matter particles with masses from 50 to $1000 \mathrm{GeV}$, the sensitivities on the self-annihilation cross section set by ANTARES and IceCube are comparable, making this mass range particularly interesting for a joint analysis. Dark matter self-annihilation through the $\tau^{+} \tau^{-}, \mu^{+} \mu^{-}, b \bar{b}$, and $W^{+} W^{-}$channels is considered for both the Navarro-Frenk-White and Burkert halo profiles. In the combination of 2101.6 days of ANTARES data and 1007 days of IceCube data, no excess over the expected background is observed. Limits on the thermally averaged dark matter annihilation cross section $\left\langle\sigma_{A} v\right\rangle$ are set. These limits present an improvement of up to a factor of 2 in the studied dark matter mass range with respect to the individual limits published by both collaborations. When considering dark matter particles with a mass of $200 \mathrm{GeV}$ annihilating through the $\tau^{+} \tau^{-}$channel, the value obtained for the limit is $7.44 \times 10^{-24} \mathrm{~cm}^{3} \mathrm{~s}^{-1}$ for the Navarro-Frenk-White halo profile. For the purpose of this joint analysis, the model parameters and the likelihood are unified, providing a benchmark for forthcoming dark matter searches performed by neutrino telescopes.

DOI: $10.1103 /$ PhysRevD.102.082002

\footnotetext{
*Also at Università di Padova, I-35131 Padova, Italy.

${ }^{\dagger}$ Also at National Research Nuclear University, Moscow Engineering Physics Institute (MEPhI), Moscow 115409, Russia.

Earthquake Research Institute, University of Tokyo, Bunkyo, Tokyo 113-0032, Japan.
}

\section{INTRODUCTION}

Dark matter was first postulated in the 1930s and its existence has been established by a wealth of astrophysical as well as cosmological observations, on both Galactic and extragalactic scales $[1,2]$. Nevertheless, the nature of dark 
matter remains largely unknown and a variety of theoretical models are considered in order to solve this mystery [3]. A common hypothesis assumes dark matter to be composed of (yet unobserved) weakly interactive massive particles (WIMPs) [4]. Searches for dark matter are typically carried out in three different ways: direct detection of nuclear recoil from WIMP-nucleus interactions [5], dark matter production in particle accelerators [6], and indirect searches [7-9]. When annihilating or decaying, dark matter particles are expected to produce Standard Model particles. These will eventually yield stable charged particles present in the cosmic radiations, as well as neutrinos and $\gamma$ rays. Indirect searches look for these messengers, which can be detected by space- or ground-based observatories.

Observations of the kinematics of stars and $\mathrm{N}$-body simulations suggest that galaxies and galaxy clusters are embedded in dark matter halos, with an increased density towards the center $[10,11]$. In addition, dark matter particles are expected to accumulate gravitationally at the center of massive objects, such as the Earth [12,13] and the Sun [14-16], after losing energy via scattering. The enhanced concentration of dark matter at the center of these objects would favor their annihilation into secondary particles, making massive objects good targets for indirect searches.

The analysis presented in this paper consists in a search for neutrinos from dark matter self-annihilation in the center of the Milky Way. In this paper, the term "neutrino" refers to $\nu+\bar{\nu}$ since the events generated by neutrinos and antineutrinos are seen indistinguishably in the two detectors considered. Corresponding limits on the thermally averaged annihilation cross section, $\left\langle\sigma_{A} v\right\rangle$, have already been set by the ANTARES and IceCube collaborations [17-21]. Both neutrino telescopes are optimized for the detection of highenergy neutrinos $(\sim 1 \mathrm{TeV})$. For dark matter masses ranging from 50 to $1000 \mathrm{GeV}$, the limits obtained by the two telescopes are comparable, which makes this region interesting for a joint analysis. By combining the data sets of both experiments, the goal is to improve the detection potential in this particular mass range. In order to perform this combined search, an important aspect was to identify the differences in the methods used by the two collaborations and to reconcile them.

This paper is structured as follows. The expected neutrino flux from dark matter annihilation is discussed in Sec. II. In Sec. III the ANTARES and IceCube neutrino detectors are presented. Section IV gives an overview of the data sets used for the combined search. The analysis method is introduced in Sec. V. In Sec. VI the systematic uncertainties are addressed. Finally, the results are shown and discussed in Sec. VII.

\section{INDIRECT DARK MATTER SEARCH WITH NEUTRINOS}

The expected differential flux of secondary neutrinos from dark matter self-annihilation in the Galactic Center is defined following Ref. [22]:

$$
\frac{\mathrm{d} \phi_{\nu}}{\mathrm{d} E_{\nu}}=\frac{1}{4 \pi} \frac{\left\langle\sigma_{A} v\right\rangle}{2 m_{\mathrm{DM}}^{2}} \frac{\mathrm{d} N_{\nu}}{\mathrm{d} E_{\nu}} J
$$

where $\left\langle\sigma_{A} v\right\rangle$ is the thermally averaged self-annihilation cross section, $m_{\mathrm{DM}}$ is the mass of the dark matter particle, and $\mathrm{d} N_{\nu} / \mathrm{d} E_{\nu}$ is the differential number of neutrinos per annihilating dark matter pair. The factor $1 / 4 \pi$ arises from the assumed spherical symmetry of the dark matter selfannihilation. The $J$-Factor is expressed as

$$
J=\int_{\Delta \Omega} \mathrm{d} \Omega(\Psi) \int_{\text {l.o.s. }} \rho_{\mathrm{DM}}^{2}(r(l, \Psi)) \mathrm{d} l
$$

and is defined as the integral over the solid angle $\Delta \Omega$ of the squared dark matter density evaluated along the line of sight (l.o.s.). The $J$-Factor depends on the opening angle to the Galactic Center, $\Psi$. The squared dark matter mass and dark matter density, as well as the factor $1 / 2$, result from the fact that two dark matter particles are needed for each annihilation.

The density distribution of dark matter in galaxies as a function of the distance $r$ to the Galactic Center can be parametrized by an extension of the Zhao profile [23]:

$$
\rho_{\mathrm{DM}}(r)=\frac{\rho_{0}}{\left(\delta+\frac{r}{r_{\mathrm{s}}}\right)^{\gamma} \cdot\left[1+\left(\frac{r}{r_{\mathrm{s}}}\right)^{\alpha}\right]^{(\beta-\gamma) / \alpha}} .
$$

Both the normalization density $\rho_{0}$ and the scale radius $r_{s}$ have to be evaluated for each galaxy. Both the ANTARES and IceCube analyses took values for these free model parameters from Ref. [24]. For consistency reasons, these values are also used for the combined search (see Table I). Since the $J$-Factor depends on the dark matter density used, we consider two dark matter halo models to account for this uncertainty. Both of them are described by Eq. (3), where the dimensionless parameters $(\alpha, \beta, \gamma, \delta)$ take the values $(1,3,1,0)$ for the Navarro-Frenk-White (NFW) profile [25] and $(2,3,1,1)$ for the Burkert profile [26]. While the two models differ by orders of magnitude close to the Galactic Center, they become rather similar outside the solar circle, $R_{\mathrm{sc}}=8.5 \mathrm{kpc}$, in agreement with uncertainty estimations from galactic rotation curves [27]. The resulting dark matter densities as a function of $r$ are shown in the left panel of Fig. 1 for both halo profiles.

Along with the spatial distribution of dark matter, given by the $J$-Factor, the spectra of secondary particles from dark matter annihilation is also a necessary theoretical input

TABLE I. Parameters of the dark matter halo profiles for the Milky Way taken from Ref. [24].

\begin{tabular}{lccc}
\hline \hline Parameters & Units & NFW & Burkert \\
\hline$\rho_{0}$ & $10^{7} M_{\odot} / \mathrm{kpc}^{3}$ & 1.4 & 4.1 \\
$r_{s}$ & $\mathrm{kpc}$ & 16.1 & 9.3 \\
\hline \hline
\end{tabular}


for this analysis. In our effort to combine the methods of both experiments, we found differences in the energy spectra used for previous analyses. While the spectra known as PPPC4 tables [28] were used by ANTARES, IceCube used spectra computed directly with PYTHIA [29]. For the purpose of the combined analysis, it was imperative to use the same spectra for both detectors. The PPPC4 tables are preferred as they take electroweak corrections into account. As a result, we noticed variations of up to $25 \%$ of the IceCube-only limits computed with the PPPC4 spectra when compared to the limits obtained with the previously used PYTHIA spectra. We consider dark matter annihilating through four self-annihilation channels. A $100 \%$ branching ratio to $W^{+} W^{-}, \tau^{+} \tau^{-}, \mu^{+} \mu^{-}$, or $b \bar{b}$ is assumed. The corresponding muon neutrino spectra at Earth for every annihilation process, $\mathrm{d} N_{\nu} / \mathrm{d} E_{\nu}$, are shown in the right panel of Fig. 1 for a dark matter mass of $100 \mathrm{GeV}$.

This analysis is sensitive to any dark matter candidate self-annihilating to Standard Model particles and leading to the production of neutrinos through the four channels studied. Throughout this work, dark matter masses ranging from 50 to $1000 \mathrm{GeV}$ are considered.

\section{DETECTORS}

Given the small interaction cross section of neutrinos, a large volume of target material is required for the neutrino detection. For Cherenkov detectors, such as ANTARES and IceCube, this was achieved by installing photomultiplier tubes (PMTs) in a transparent natural medium. These photosensors then record the Cherenkov emission induced by secondary charged particles produced by the interaction of neutrinos in the surrounding environment.

ANTARES is an underwater neutrino telescope deployed in the Mediterranean Sea, $40 \mathrm{~km}$ offshore from Toulon, France at coordinates $42^{\circ} 48^{\prime} \mathrm{N}, 6^{\circ} 10^{\prime} \mathrm{E}$ [30]. The detector is composed of 12 vertical detection lines, horizontally spaced by $70 \mathrm{~m}$. Each string holds 25 storeys of three

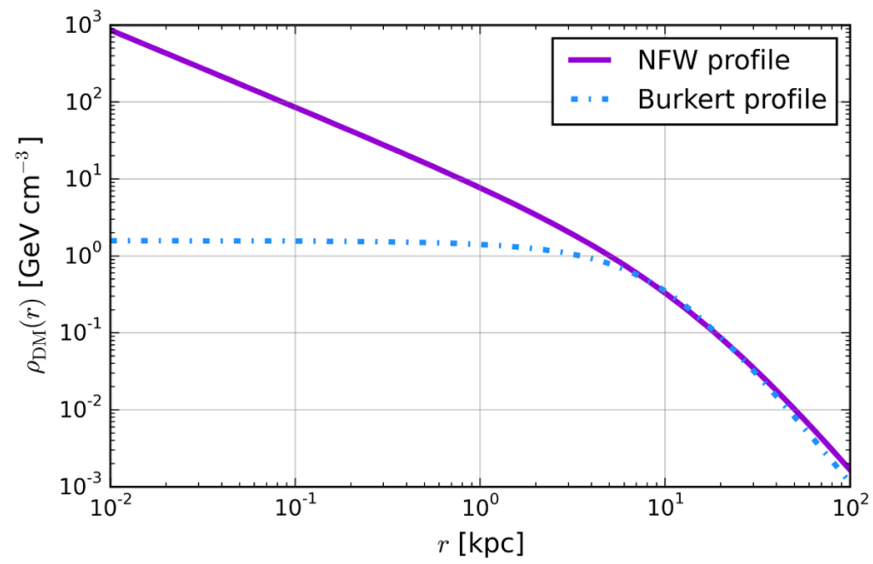

photodetectors separated vertically by $14.5 \mathrm{~m}$. The strings are anchored to the seabed at a depth of $2475 \mathrm{~m}$, covering a volume of more than $0.01 \mathrm{~km}^{3}$.

IceCube is a cubic-kilometer neutrino telescope located at the geographic South Pole [31]. The detector consists of 5160 PMTs attached to vertical strings disseminated in 86 boreholes [32], between depths of 1450 to $2500 \mathrm{~m}$. The IceCube array is composed of 86 strings instrumented with 60 digital optical modules (DOMs). Among them, 78 strings are arranged on a hexagonal grid with a spacing of $125 \mathrm{~m}$, with a vertical separation of $17 \mathrm{~m}$ between each DOM. The eight remaining strings are deployed more compactly at the center of the array, forming the DeepCore subdetector [33]. A horizontal distance of $72 \mathrm{~m}$ separates the DeepCore strings with a vertical spacing of $7 \mathrm{~m}$ between each DOM. The fiducial volume of DeepCore forms a $125 \mathrm{~m}$ radius by $350 \mathrm{~m}$ long cylinder, which includes seven regular IceCube strings.

\section{EVENT SELECTION}

This joint analysis makes use of individual data sets which were designed for previous analyses of the corresponding collaborations. Both samples are optimized to search for dark matter in the Galactic Center. Considering the different scales and locations of the two detectors, distinct methods were used to reduce the background. The main backgrounds of neutrino telescopes consist of atmospheric muons and neutrinos produced by the interaction of cosmic rays with nuclei in the upper atmosphere. The contribution from atmospheric muons is 6 orders of magnitude larger than the background from atmospheric neutrinos. However, in the up-going direction, muons are suppressed as they are filtered out by the Earth.

The Galactic Center is located in the Southern Hemisphere, at declination $\delta_{\mathrm{GC}} \sim-29.01^{\circ}$. Since declinations between $0^{\circ}$ and $-90^{\circ}$ are always above the horizon of IceCube, events coming from the Galactic Center are seen

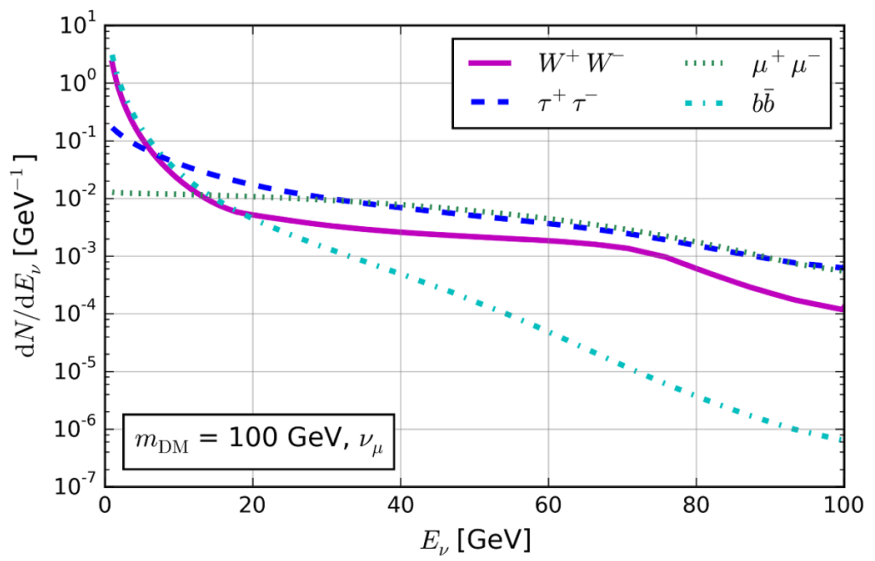

FIG. 1. Left: dark matter density $\rho_{\mathrm{DM}}(r)$ as a function of the radial distance to the Galactic Center $r$ for the NFW and Burkert profiles. Right: muon neutrino spectra at Earth for a dark matter mass of $100 \mathrm{GeV}$ and the four self-annihilation channels. 
as down-going events in the detector. Therefore, we consider a smaller fiducial volume for this analysis, since the outer part of the detector is used as a veto to reject atmospheric muons. The effective volume is reduced to the eight DeepCore strings and the seven surrounding IceCube strings. In addition, only DOMs with depths between 2140 and $2420 \mathrm{~m}$ are considered. Hence, the resulting effective volume for IceCube is about $0.015 \mathrm{~km}^{3}$, which is comparable to the ANTARES instrumented volume. Unlike IceCube, ANTARES does not have a fixed view of the Galactic Center in local coordinates. Hence, declinations below $-47^{\circ}$ are favored since they are always seen as upgoing in the ANTARES detector, while events with declinations between $-47^{\circ}$ and $47^{\circ}$ are below the horizon for only a part of the sidereal day. As a result, ANTARES is able to view the Galactic Center about $75 \%$ of the time and no instrumental veto is required for this analysis.

The ANTARES data set consists of events recorded over 9 years between 2007 and 2015, resulting in an effective livetime of 2101.6 days. This sample is composed of upgoing track-like events and was optimized for a previous dark matter search based on the same data set [17]. According to the number of strings with triggered PMTs, two different reconstruction algorithms are used. The single-line reconstruction (QFit) [34], which is optimized for energies below $100 \mathrm{GeV}$, can reconstruct only the zenith angle of the events. At higher neutrino energies, the multi-line algorithm ( $\lambda$ Fit) is used [35] since PMTs from more than one string are likely to be triggered. Both algorithms are characterized by a parameter representing the quality of the reconstructed track. The final selection results in 1077 reconstructed neutrino events for QFit and 15651 events for $\lambda$ Fit. Since these cuts strongly favor the reconstruction of muon tracks produced in the chargedcurrent interaction of muon neutrinos, only neutrinos of this flavor are considered.

For IceCube, a data sample thoroughly described in Ref. [19] is used. That selection consists of events recorded from 2012 to 2015 with the 86-string configuration, for a total livetime of 1007 days. The purpose was to select tracklike events starting within the detector volume. Such events originate mainly from the charged-current interactions of muon neutrinos within the detector. Unlike ANTARES, this event selection does not completely reject non-muon neutrinos. Therefore, electron or tau neutrinos with similar topology remain in the sample. The final selection results in a total of 22622 events.

\section{ANALYSIS PROCEDURE}

A binned likelihood method is applied in order to search for an excess of signal neutrinos from the Galactic Center. In this approach, the distribution of the data is compared to what is expected from the background and signal distributions for given combinations of halo profile, dark matter mass, and annihilation channel. The information about the shape of the signal and background is contained in probability density functions (PDFs). Likelihood functions are defined for each experiment, with PDFs built differently for ANTARES and IceCube.

The ANTARES PDFs represent the angular distance of each event from the source. For QFit, we use 28 bins in $\Delta \cos (\theta)=\cos \left(\theta_{\mathrm{GC}}\right)-\cos \left(\theta_{\text {event }}\right)$ from -1 to 0.14 , where $\cos \left(\theta_{\text {event }}\right)$ is the zenith of the reconstructed event track and $\cos \left(\theta_{\mathrm{GC}}\right)$ represents the zenith position of the Galactic Center at the time of the event (see top panel of Fig. 2). For $\lambda$ Fit, we consider 15 bins in $\Psi$ ranging from $0^{\circ}$ to $30^{\circ}$, where $\Psi$ is the space angle between the Galactic Center and the event track (see bottom panel of Fig. 2). In the case of IceCube, two-dimensional distributions are used (see Fig. 3). The binning consists of six bins in declination ranging from -1 to $1 \mathrm{rad}$ and ten bins in right ascension (RA) covering the range from $-\pi$ to $\pi \mathrm{rad}$.

For both experiments, the signal PDFs are estimated from generic samples of simulated neutrinos, which are then weighted with the source morphology and the neutrino spectrum for each halo profile, dark matter mass, and
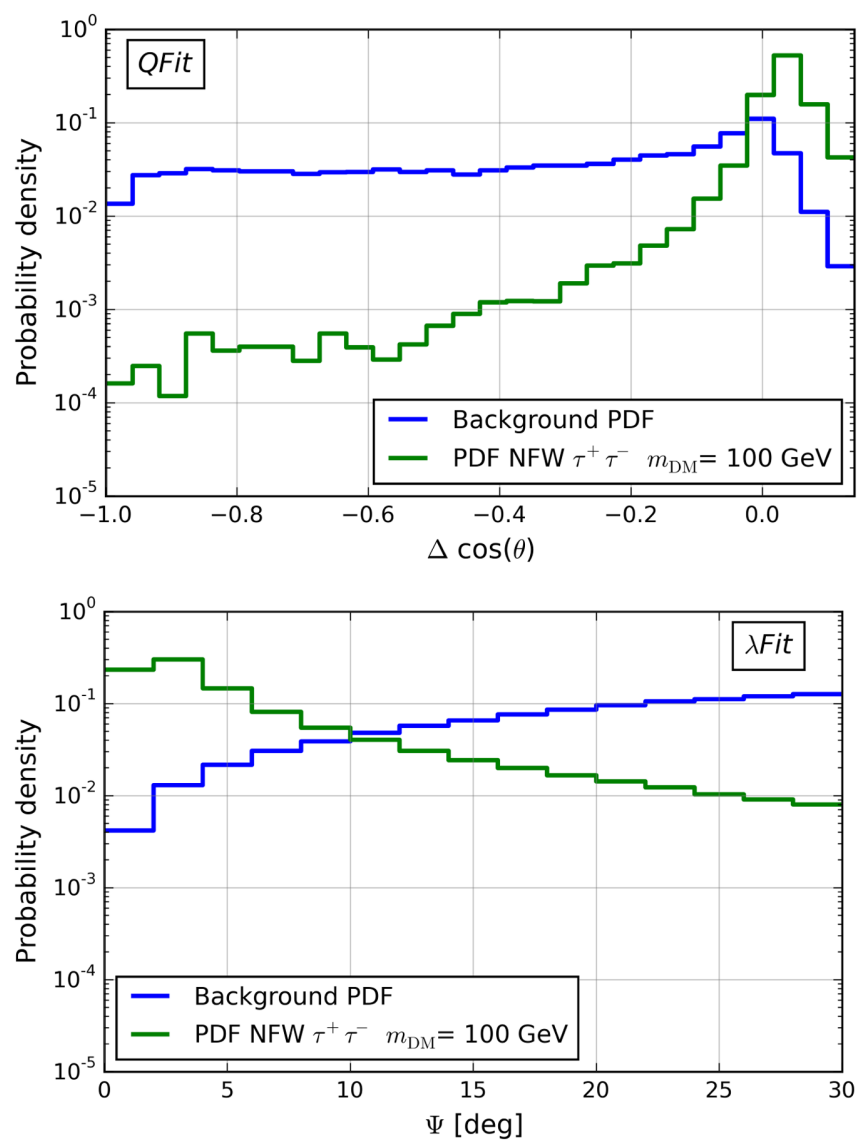

FIG. 2. Top: ANTARES PDFs for the QFit reconstruction. Bottom: ANTARES PDFs for the $\lambda$ Fit reconstruction. Both histograms show the background (blue) and signal (green) PDFs for the $\tau^{+} \tau^{-}$annihilation channel and NFW profile, assuming $m_{\mathrm{DM}}=100 \mathrm{GeV}$. 

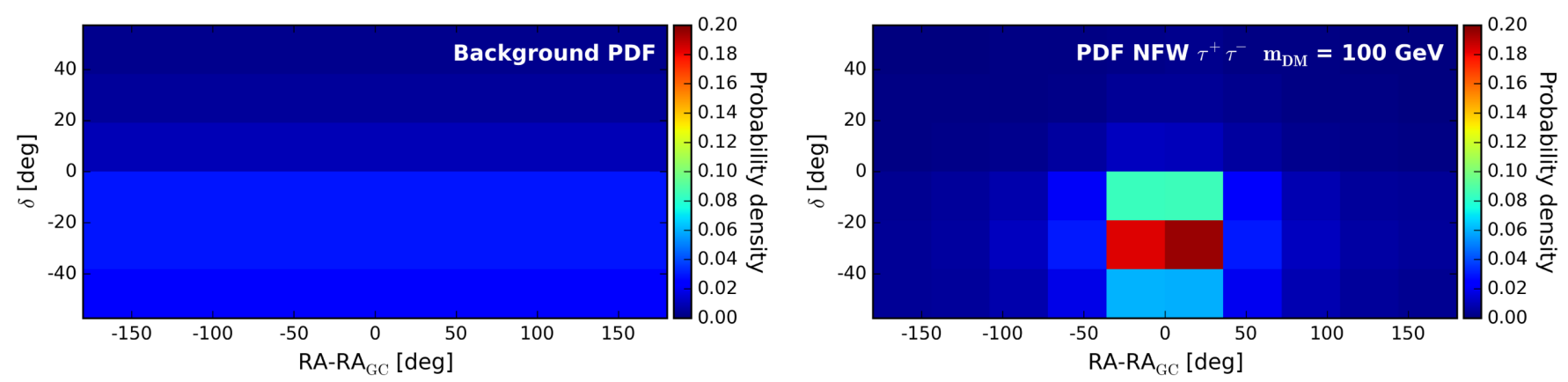

FIG. 3. Left: IceCube background PDF obtained from data scrambled in RA, where the color scale expresses the probability density. Right: IceCube signal PDF for the $\tau^{+} \tau^{-}$channel and $m_{\mathrm{DM}}=100 \mathrm{GeV}$ assuming the NFW profile.

annihilation channel. Assuming uniformity of the background in RA, the IceCube background PDF is determined by scrambling the data in RA and subtracting the expected signal. For ANTARES, the $\lambda$ Fit background PDF is also determined from experimental data scrambled in RA, while the QFit background PDF is obtained by scrambling the arrival time of the events.

Any given event distribution $f^{i}(\mu)$ can be expressed as a superposition of the signal $f_{\mathrm{s}}^{i}$ and background $f_{\mathrm{bg}}^{i}$ PDFs:

$$
f^{i}(\mu)=\mu f_{\mathrm{s}}^{i}+(1-\mu) f_{\mathrm{bg}}^{i},
$$

where $\mu \in[0,1]$ is the fraction of signal events assumed to be present in the total sample.

The likelihood is defined as the product of the Poisson probabilities to observe $n_{\mathrm{obs}}^{i}$ events in a particular bin $i$ :

$$
\mathcal{L}(\mu)=\prod_{i=\min }^{\max } \frac{\left(n_{\mathrm{obs}}^{\mathrm{tot}} f^{i}(\mu)\right)^{n_{\mathrm{obs}}^{i}}}{n_{\mathrm{obs}}^{i} !} e^{-n_{\mathrm{obs}}^{\mathrm{tot}} f^{i}(\mu)}
$$

The number of observed events in a bin $i, n_{\mathrm{obs}}^{i}$, is compared to the expected number of events in that particular bin, given the total number of events in the data, $n_{\text {obs }}^{\text {tot }}$, times the fraction of events within a specific bin, $f^{i}(\mu)$, for a given value of $\mu$.

Once defined for ANTARES and IceCube separately, the likelihoods are merged into a single combined likelihood defined as

$$
\mathcal{L}_{\mathrm{comb}}(\mu)=\prod_{k=A, I} \mathcal{L}_{k}\left(w_{k} \cdot \mu\right),
$$

where the index $k=(A, I)$ refers to ANTARES and IceCube, respectively. Since the signal acceptances $\eta_{\text {sig }}^{k}$ for a given dark matter signal (mass, annihilation channel, and halo profile) are different for the two experiments, the signal fraction is weighted with a relative weight, $w_{k}$. This weight represents the relative signal acceptance of each experiment with respect to the contribution from the total event sample:

$$
w_{k}=\frac{\eta_{\mathrm{sig}}^{k} / \eta_{\mathrm{sig}}}{N_{\mathrm{tot}}^{k} / N_{\mathrm{tot}}}
$$

where $N_{\text {tot }}$ denotes the total number of background events and is obtained by summing $N_{\text {tot }}^{A}$ and $N_{\text {tot }}^{I}$. The total signal acceptance $\eta_{\text {sig }}$ is defined as the sum of the individual signal acceptances $\eta_{\text {sig }}^{k}$, which we define as

$$
\eta_{\text {sig }}^{k}=\frac{1}{8 \pi} \frac{J}{T_{\text {live }}^{k}} m_{\mathrm{DM}}^{2} \int A_{\text {eff }}^{k}(E) \frac{\mathrm{d} N_{\nu}}{\mathrm{d} E_{\nu}} \mathrm{d} E
$$

where $T_{\text {live }}^{k}$ is the experiment livetime and $A_{\text {eff }}^{k}$ is the effective area of the detector. The signal acceptances are computed for each combination of dark matter mass, annihilation channel, and halo profile. The effective area, computed using Monte Carlo simulations, depends on several factors, such as the neutrino cross section, the range of secondary particles, the detector efficiencies, and the selection criteria for each sample. A comparison of the effective area of the ANTARES and IceCube samples is shown in Fig. 4 for declinations between $\delta_{\mathrm{GC}}-30^{\circ}$ and

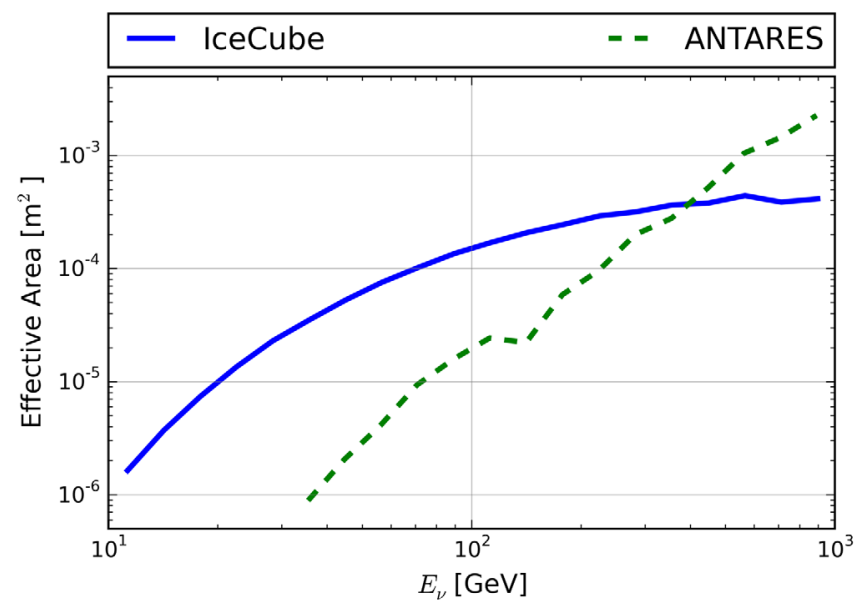

FIG. 4. Comparison of the effective area of the ANTARES and IceCube samples as a function of the neutrino energy for events with declination $\delta \in\left[\delta_{\mathrm{GC}}-30^{\circ}, \delta_{\mathrm{GC}}+30^{\circ}\right]$. 
$\delta_{\mathrm{GC}}+30^{\circ}$. The different behavior of the two curves can be explained by the fact that, while IceCube is limited to its vetoed fiducial volume in order to limit the background from atmospheric muons, ANTARES does not need to restrict itself to events starting within the detector volume. Therefore, the IceCube effective area hits a plateau at higher energies, while ANTARES can extend its fiducial volume beyond the detector boundaries. For ANTARES, the transition between the QFit and $\lambda$ Fit reconstructions is visible around $130 \mathrm{GeV}$.

With this likelihood method, we can obtain the best estimate of the signal fraction, $\mu_{\text {best }}$, which is the value of $\mu$ that maximizes the likelihood, $\mathcal{L}(\mu)$. In order to evaluate the sensitivity of this analysis, we generate 100000 pseudoexperiments sampled from the background-only PDF. For each of these pseudoexperiments, we compute the upper limit at the $90 \%$ confidence level (C.L.), $\mu_{90}$, according to the unified approach of Feldman and Cousins [36]. The final sensitivity, $\hat{\mu}_{90}$, is defined as the median value of these upper limits. The $\mu_{90}$ distribution of the pseudoexperiments is also used to determine the statistical uncertainty of the sensitivity, which we express in terms of $1 \sigma$ and $2 \sigma$ uncertainties.

The same method is used to determine $\mu_{\text {best }}$ for the unblinded data. If the obtained value is consistent with the background-only hypothesis, the corresponding upper limit on the signal fraction, $\mu_{90}$, is computed. We can then deduce the limit on the dark matter self-annihilation cross section using the relation

$$
\left\langle\sigma_{\mathrm{A}} v\right\rangle=\frac{\mu_{90} N_{\text {tot }}}{\eta_{\text {sig }}}
$$

for a given dark matter mass, annihilation channel, and halo profile.

\section{SYSTEMATIC UNCERTAINTIES}

The sources of uncertainties can be split into theoretical and detector-related systematic uncertainties. Since the background PDFs are obtained from data for both experiments, systematic effects were only studied for the signal simulation.

For ANTARES, the uncertainty on the track direction is the dominant systematic uncertainty. To account for this, the approach used in previous ANTARES point-source searches is applied. The determination of track parameters relies on the time resolution of the detector units, affected by the PMT transit time spread, errors in the calibration of the timing system, and possible spatial misalignment of the detector lines. As reported in Ref. [35], these uncertainties overall affect the angular resolution for tracks by about $15 \%$. This uncertainty is implemented in the analysis by smearing the signal PDFs by $15 \%$.

Similarly, the dominant source of systematic uncertainty of the IceCube detector results from the uncertainty on the angular resolution, which is affected by the modeling of the ice properties and the photon detection efficiency of the DOMs. These effects were studied using Monte Carlo simulations for which variations of $\pm 1 \sigma$ on the baseline set of values were applied. This results in a 5-15\% uncertainty from the optical properties of the ice, where the scattering and absorption lengths are modified. The optical properties of the hole ice are different than the bulk ice. Due to the presence of impurities, the scattering length of the ice in the drilling holes is shorter. The treatment of the uncertainty on the scattering length results in a worsening of the sensitivity by $25-30 \%$ when increasing the scattering length considered for the hole ice. Reciprocally, the sensitivity improves by $5-10 \%$ when considering a shortening of the scattering length. The uncertainty on the photon detection efficiency of the DOMs affects the sensitivity by improving or worsening it by $5-40 \%$. We add in quadrature the different systematic contributions to obtain the total uncertainty, assuming all systematic uncertainties to be independent. These systematic uncertainties are included in the final results by conservatively reducing the IceCube signal acceptance $\eta_{\text {sig }}^{\mathrm{I}}$ by $38 \%$.

However, astrophysical uncertainties on the dark matter halo model parameters prevail over the systematic uncertainties mentioned above. For instance, alternative estimates of these model parameters for the NFW halo profile [37] can affect the limit by up to a factor of 1.5 . To account for uncertainties linked to the dark matter halo models, we present limits for both the NFW and Burkert profiles. The impact of the halo model choice can be seen in Fig. 5, where the limits for the NFW and Burkert profiles are presented.

\section{RESULTS AND DISCUSSION}

This joint analysis is conducted with data collected by the ANTARES and IceCube neutrino telescopes during periods of 9 and 3 years, respectively. By combining the data samples at the likelihood level, we find no significant excess of neutrinos in the direction of the Galactic Center. We present limits on the thermally averaged dark matter self-annihilation cross section $\left\langle\sigma_{A} v\right\rangle$. The values obtained for all dark matter masses and annihilation channels can be found in Tables II and III for the NFW and Burkert profiles, respectively, with parameters from Table I. The 90\% C.L. combined limits are presented in Fig. 5 for all selfannihilation channels considered, assuming both the NFW (top) and Burkert (bottom) halo profiles. The dissimilar behavior of these limits for the NFW and Burkert profiles originates from the two event reconstructions used for ANTARES. The transition point from QFit to $\lambda$ Fit depends on the balance between the number of reconstructed events and the quality of the reconstruction. The better angular resolution provided by $\lambda$ Fit is more beneficial when considering a "cuspy" dark matter halo profile such as the NFW profile. Therefore, the transition 

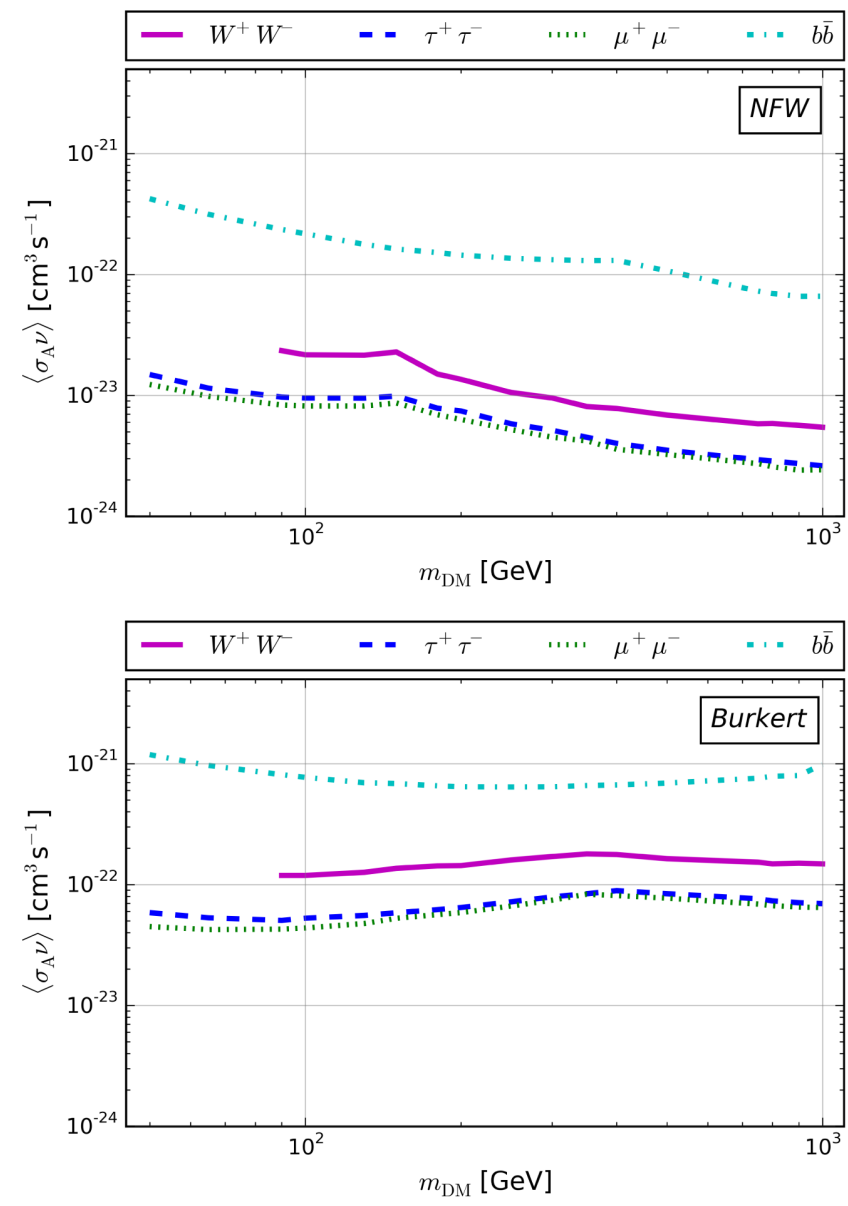

FIG. 5. Combined $90 \%$ C.L. limits on the thermally averaged dark matter annihilation cross section as a function of the dark matter mass for the NFW (top) and Burkert (bottom) halo profiles. All annihilation channels considered in this analysis are presented $\left(b \bar{b}, \tau^{+} \tau^{-}, \mu^{+} \mu^{-}, W^{+} W^{-}\right)$.

between the two reconstructions happens at a lower dark matter mass for the NFW profile.

In Fig. 6 we present the combined limit obtained for the $\tau^{+} \tau^{-}$channel and the NFW profile alongside the previous ANTARES and IceCube limits. The present analysis uses the data sets developed for these individual searches. When compared to the IceCube and ANTARES standalone limits, the combined limit is better by up to a factor of 2 in the dark matter mass range considered, i.e., between 50 and $1000 \mathrm{GeV}$. An enhancement of the limit can also be seen for the other dark matter annihilation channel and halo profile combinations presented in Fig. 5, with an exception for the $b \bar{b}$ channel when considering the Burkert profile. For this particular case, the combined limit is dominated by IceCube, which has a better signal acceptance than ANTARES for the entire mass range due to the very soft spectrum. In addition to the improvement due to the combination of the two data sets, a difference between the ANTARES limit and the combined limit is also noticeable for dark matter masses close to $1 \mathrm{TeV}$, where
TABLE II. 90\% C.L. upper limits on the thermally averaged self-annihilation cross section for the NFW profile.

\begin{tabular}{lcccc}
\hline \hline & \multicolumn{4}{c}{$\left\langle\sigma_{A} v\right\rangle\left[10^{-24} \mathrm{~cm}^{3} \mathrm{~s}^{-1}\right]$} \\
\cline { 2 - 5 }$m_{\mathrm{DM}}[\mathrm{GeV}]$ & $b \bar{b}$ & $\tau^{+} \tau^{-}$ & $\mu^{+} \mu^{-}$ & $W^{+} W^{-}$ \\
\hline 50 & 424 & 14.9 & 12.3 & $\ldots$ \\
65 & 315 & 11.5 & 9.8 & $\ldots$ \\
90 & 236 & 9.7 & 8.3 & 23.5 \\
100 & 217 & 9.5 & 8.2 & 21.7 \\
130 & 177 & 9.5 & 8.2 & 21.5 \\
150 & 162 & 9.9 & 8.7 & 22.9 \\
180 & 157 & 7.9 & 6.9 & 15.0 \\
200 & 144 & 7.4 & 6.3 & 13.6 \\
250 & 136 & 5.8 & 5.2 & 10.6 \\
300 & 132 & 5.2 & 4.5 & 9.5 \\
350 & 130 & 4.5 & 4.2 & 8.1 \\
400 & 131 & 4.0 & 3.6 & 7.8 \\
500 & 107 & 3.5 & 3.3 & 6.9 \\
750 & 72.9 & 2.9 & 2.7 & 5.8 \\
800 & 69.7 & 2.9 & 2.6 & 5.9 \\
900 & 66.0 & 2.7 & 2.4 & 5.7 \\
1000 & 66.1 & 2.6 & 2.4 & 5.5 \\
\hline \hline
\end{tabular}

the contribution from IceCube is expected to be negligible. This divergence results mainly from the way underfluctuations are treated by this analysis and the previous ANTARES search. When obtaining limits with lower values than sensitivities, sensitivities were labeled as limits for the previous ANTARES analysis while limits remain unchanged for our combined search. Furthermore, the ANTARES analysis used the Neyman approach [38] with slightly different PDFs for the $\lambda$ Fit reconstruction. The importance of these changes can be seen in Fig. 7, where

TABLE III. 90\% C.L. upper limits on the thermally averaged self-annihilation cross section for the Burkert profile.

\begin{tabular}{lcccc}
\hline \hline & \multicolumn{4}{c}{$\left\langle\sigma_{A} v\right\rangle\left[10^{-23} \mathrm{~cm}^{3} \mathrm{~s}^{-1}\right]$} \\
\cline { 2 - 5 }$m_{\mathrm{DM}}[\mathrm{GeV}]$ & $b \bar{b}$ & $\tau^{+} \tau^{-}$ & $\mu^{+} \mu^{-}$ & $W^{+} W^{-}$ \\
\hline 50 & 118 & 5.9 & 4.5 & $\ldots$ \\
65 & 96.8 & 5.3 & 4.2 & $\ldots$ \\
90 & 81.2 & 5.1 & 4.3 & 11.9 \\
100 & 77.1 & 5.3 & 4.4 & 11.9 \\
130 & 69.6 & 5.6 & 4.8 & 12.6 \\
150 & 68.6 & 5.9 & 5.3 & 13.6 \\
180 & 65.7 & 6.2 & 5.7 & 14.3 \\
200 & 64.5 & 6.5 & 5.9 & 14.4 \\
250 & 64.2 & 7.2 & 6.7 & 15.9 \\
300 & 64.4 & 7.9 & 7.4 & 17.1 \\
350 & 65.9 & 8.4 & 8.3 & 17.9 \\
400 & 66.8 & 8.9 & 8.1 & 17.8 \\
500 & 69.1 & 8.4 & 7.7 & 16.4 \\
750 & 75.9 & 7.7 & 6.9 & 15.3 \\
800 & 78.7 & 7.3 & 6.7 & 14.8 \\
900 & 79.8 & 7.1 & 6.5 & 15.0 \\
1000 & 98.7 & 7.0 & 6.5 & 14.8 \\
\hline \hline
\end{tabular}




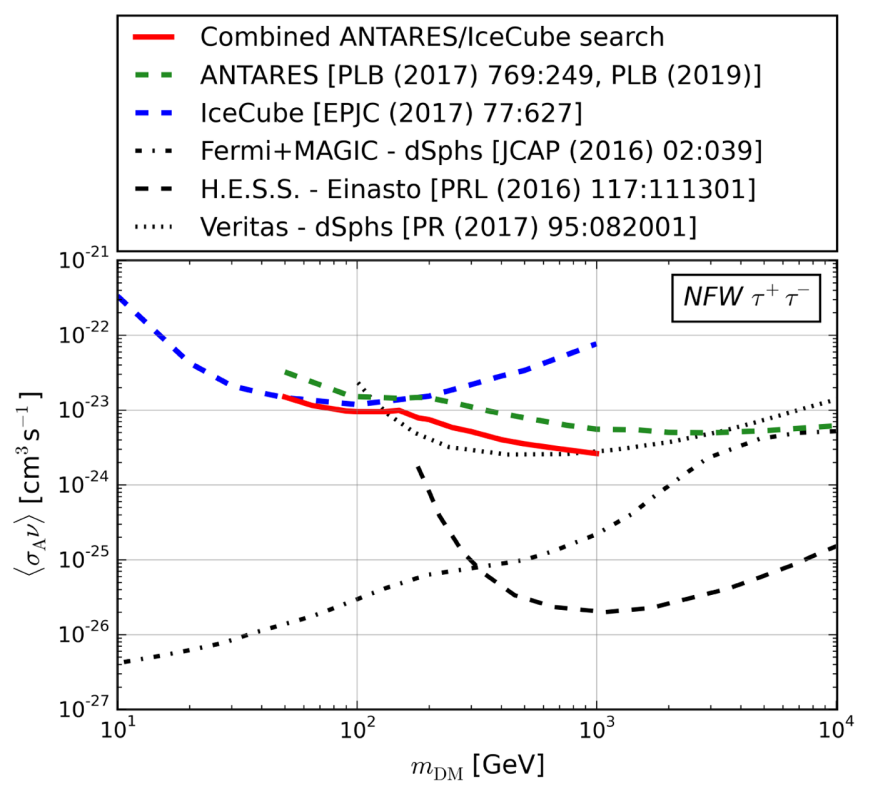

FIG. 6. 90\% C.L. upper limit on the thermally averaged dark matter annihilation cross section $\left\langle\sigma_{A} v\right\rangle$ obtained for the combined analysis as a function of the dark matter mass $m_{\mathrm{DM}}$ assuming the NFW halo profile for the $\tau^{+} \tau^{-}$annihilation channel. The limits from IceCube [19], ANTARES [17], VERITAS [8], Fermi + MAGIC [9], and H.E.S.S. [7] are also shown.

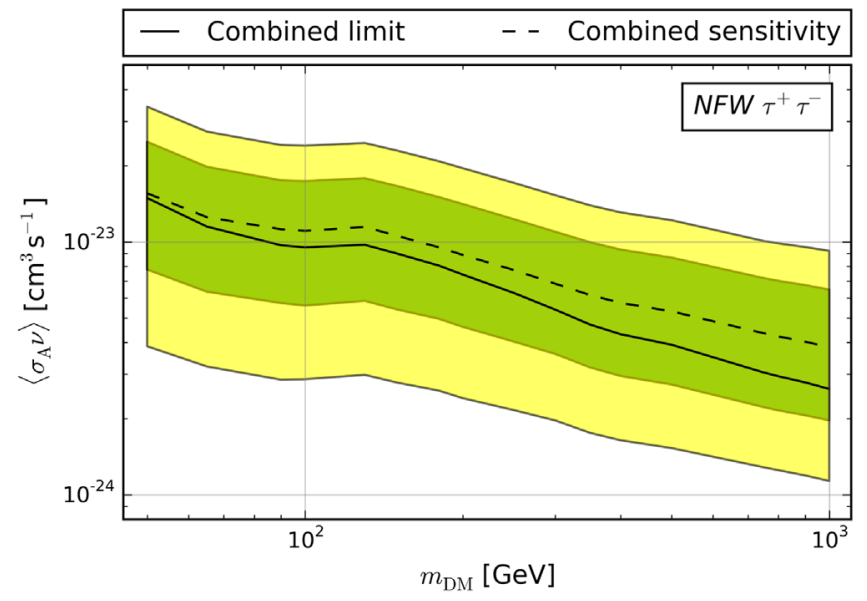

FIG. 7. Comparison of the $90 \%$ C.L. combined limit (solid line) and sensitivity (dashed line) for the NFW halo profile and the $\tau^{+} \tau^{-}$annihilation channel, along with the expected $1 \sigma$ (green) and $2 \sigma$ (yellow) bands around the expected median sensitivity.

the limit for dark matter annihilation into $\tau^{+} \tau^{-}$for the NFW profile is shown alongside the sensitivity. These results are also compared with current limits obtained with $\gamma$-ray telescopes from searches of photons produced in the self-annihilation of dark matter into $\tau^{+} \tau^{-}$(see Fig. 6). Gamma-ray limits are still several orders of magnitude better for this particular channel, although it needs to be noted that the VERITAS [8] and combined Fermi + MAGIC limits [9] were obtained from the study of dwarf spheroidal galaxies (dSphs), while the other limits presented are for the Galactic Center. Note as well that the H.E.S.S. limit was obtained assuming the Einasto halo profile [7]. Although both the NFW and Einasto halo profiles assume a high dark matter density at the center of the Galaxy, the difference between the profiles is nonnegligible in the central region. Moreover, there is considerable freedom in the choice of halo parameters, and these choices are not made consistently between experiments. The halo parameters used in this work are conservative with respect to more optimistic values made in other analyses, and this freedom is responsible for some of the difference between the limits set by IceCube and the more stringent limits reported in Ref. [7].

\section{ACKNOWLEDGMENTS}

The authors from the ANTARES Collaboration acknowledge the financial support of the following funding agencies: Centre National de la Recherche Scientifique (CNRS), Commissariat à l'énergie atomique et aux énergies alternatives (CEA), Commission Européenne (FEDER fund and Marie Curie Program), Institut Universitaire de France (IUF), IdEx program and UnivEarthS Labex program at Sorbonne Paris Cité (ANR-10-LABX-0023 and ANR-11IDEX-0005-02), Labex OCEVU (ANR-11-LABX-0060) and the A*MIDEX project (ANR-11-IDEX-0001-02), Région Île-de-France (DIM-ACAV), Région Alsace (contrat CPER), Région Provence-Alpes-Côte d'Azur, Département du Var and Ville de La Seyne-sur-Mer, France; Bundesministerium für Bildung und Forschung (BMBF), Germany; Istituto Nazionale di Fisica Nucleare (INFN), Italy; Nederlandse organisatie voor Wetenschappelijk Onderzoek (NWO), the Netherlands; Council of the President of the Russian Federation for young scientists and leading scientific schools supporting grants, Russia; Executive Unit for Financing Higher Education, Research, Development and Innovation (UEFISCDI), Romania; Ministerio de Ciencia, Innovación, Investigación y Universidades (MCIU): Programa Estatal de Generación de Conocimiento (refs. PGC2018-096663-B-C41, -A-C42, -B-C43, -B-C44) (MCIU/FEDER), Severo Ochoa Centre of Excellence and MultiDark Consolider (MCIU), Junta de Andalucía (ref. SOMM17/6104/UGR), Generalitat Valenciana: Grisolía (ref. GRISOLIA/2018/119), Spain; Ministry of Higher Education, Scientific Research and Professional Training, Morocco. We also acknowledge the technical support of Ifremer, AIM and Foselev Marine for the sea operation and CC-IN2P3 for the computing facilities. The authors from the IceCube Collaboration gratefully acknowledge the support from the following agencies and institutions: USA-U.S. National Science Foundation-Office of Polar Programs, U.S. National Science Foundation-Physics Division, Wisconsin Alumni Research Foundation, Center for High Throughput Computing (CHTC) at the University of Wisconsin-Madison, Open Science Grid (OSG), Extreme 
Science and Engineering Discovery Environment (XSEDE), U.S. Department of Energy-National Energy Research Scientific Computing Center, Particle astrophysics research computing center at the University of Maryland, Institute for Cyber-Enabled Research at Michigan State University, and Astroparticle physics computational facility at Marquette University; Belgium-Funds for Scientific Research (FRS-FNRS and FWO), FWO Odysseus and Big Science programmes, and Belgian Federal Science Policy Office (Belspo); Germany-Bundesministerium für Bildung und Forschung (BMBF), Deutsche Forschungsgemeinschaft (DFG), Helmholtz Alliance for Astroparticle Physics (HAP), Initiative and Networking Fund of the Helmholtz Association, Deutsches Elektronen Synchrotron (DESY), and High Performance Computing cluster of the RWTH Aachen; SwedenSwedish Research Council, Swedish Polar Research
Secretariat, Swedish National Infrastructure for Computing (SNIC), and Knut and Alice Wallenberg Foundation; Australia-Australian Research Council; Canada-Natural Sciences and Engineering Research Council of Canada, Calcul Québec, Compute Ontario, Canada Foundation for Innovation, WestGrid, and Compute Canada; Denmark-Villum Fonden, Danish National Research Foundation (DNRF), Carlsberg Foundation; New Zealand-Marsden Fund; Japan—Japan Society for Promotion of Science (JSPS) and Institute for Global Prominent Research (IGPR) of Chiba University; Korea-National Research Foundation of Korea (NRF); Switzerland-Swiss National Science Foundation (SNSF); United Kingdom-Department of Physics, University of Oxford. The IceCube collaboration acknowledges the significant contributions to this manuscript from Sebastian Baur, Nadège Iovine and Sara Rebecca Gozzini.
[1] G. Bertone and D. Hooper, Rev. Mod. Phys. 90, 045002 (2018).

[2] P. A. R. Ade et al. (Planck Collaboration), Astron. Astrophys. 571, 66 (2014).

[3] G. Bertone, D. Hooper, and J. Silk, Phys. Rep. 405, 279 (2005).

[4] G. Steigman and M. S. Turner, Nucl. Phys. B253, 375 (1985).

[5] J. Liu, X. Chen, and X. Ji, Nat. Phys. 13, 212 (2017).

[6] A. Boveia and C. Doglioni, Annu. Rev. Nucl. Part. Sci. 68, 429 (2018).

[7] H. Abdallah et al. (H.E.S.S. Collaboration), Phys. Rev. Lett. 117, 111301 (2016).

[8] S. Archambault et al. (VERITAS Collaboration), Phys. Rev. D 95, 082001 (2017).

[9] M. L. Ahnen et al. (MAGIC and Fermi-LAT Collaborations), J. Cosmol. Astropart. Phys. 02 (2016) 039.

[10] B. Moore, T. Quinn, F. Governato, J. Stadel, and G. Lake, Mon. Not. R. Astron. Soc. 310, 1147 (1999).

[11] A. V. Kravtsov, A. A. Klypin, J. S. Bullock, and J. R. Primack, Astrophys. J. 502, 48 (1998).

[12] M. G. Aartsen et al. (IceCube Collaboration), Eur. Phys. J. C 77, 82 (2017).

[13] A. Albert et al. (ANTARES Collaboration), Phys. Dark Universe 16, 41 (2017).

[14] M. G. Aartsen et al. (IceCube Collaboration), Eur. Phys. J. C 77, 146 (2017).

[15] S. Adrián-Martínez et al. (ANTARES Collaboration), Phys. Lett. B 759, 69 (2016).

[16] S. Adrián-Martínez et al. (ANTARES Collaboration), J. Cosmol. Astropart. Phys. 05 (2016) 016.

[17] A. Albert et al. (ANTARES Collaboration), Phys. Lett. B 769, 249 (2017); 796, 253(E) (2019).
[18] A. Martinez et al. (ANTARES Collaboration), J. Cosmol. Astropart. Phys. 10 (2015) 068.

[19] M. G. Aartsen et al. (IceCube Collaboration), Eur. Phys. J. C 77, 627 (2017).

[20] R. Abbasi et al. (IceCube Collaboration), Phys. Rev. D 84, 022004 (2011).

[21] M. G. Aartsen et al. (IceCube Collaboration), Eur. Phys. J. C 75, 20 (2015).

[22] H. Yuksel, S. Horiuchi, J. F. Beacom, and S. Ando, Phys. Rev. D 76, 123506 (2007).

[23] J. An and H. Zhao, Mon. Not. R. Astron. Soc. 428, 2805 (2013).

[24] F. Nesti and P. Salucci, J. Cosmol. Astropart. Phys. 07 (2013) 016.

[25] J. F. Navarro, C. S. Frenk, and S. D. M. White, Astrophys. J. 462, 563 (1996).

[26] A. Burkert, Astrophys. J. 447, L25 (1995); IAU Symp. 171, 175 (1996)

[27] M. Benito, A. Cuoco, and F. Iocco, J. Cosmol. Astropart. Phys. 03 (2019) 033.

[28] M. Cirelli, G. Corcella, A. Hektor, G. Hutsi, M. Kadastik, P. Panci, M. Raidal, F. Sala, and A. Strumia, J. Cosmol. Astropart. Phys. 03 (2011) 051; 10 (2012) E01.

[29] T. Sjöstrand, P. Eden, C. Friberg, G. Miu, L. Lönnblad, S. Mrenna, and E. Norrbin, Comput. Phys. Commun. 135, 238 (2001); T. Sjöstrand, S. Mrenna, and P. Z. Skands, J. High Energy Phys. 05 (2006) 026.

[30] M. Ageron et al. (ANTARES Collaboration), Nucl. Instrum. Methods Phys. Res., Sect. A 656, 11 (2011).

[31] M. G. Aartsen et al. (IceCube Collaboration), J. Instrum. 12, P03012 (2017).

[32] R. Abbasi et al. (IceCube Collaboration), Nucl. Instrum. Methods Phys. Res., Sect. A 618, 139 (2010). 
[33] R. Abbasi et al. (IceCube Collaboration), Astropart. Phys. 35, 615 (2012).

[34] J. Aguilar et al. (ANTARES Collaboration), Astropart. Phys. 34, 652 (2011).

[35] S. Adrian-Martinez et al. (ANTARES Collaboration), Astrophys. J. 760, 53 (2012).
[36] G. J. Feldman and R. D. Cousins, Phys. Rev. D 57, 3873 (1998).

[37] P. J. McMillan, Mon. Not. R. Astron. Soc. 465, 76 (2017).

[38] J. Neyman, Phil. Trans. R. Soc. A 236, 333 (1937). 